\title{
ALGUNOS FACTORES ASOCIADOS CON LA MORTALIDAD INFANTIL EN MÉXICO
}

\author{
IRMA O. GARCÍA Y GARMA \\ El Colegio de México
}

\section{NIVELES Y TENDENCIAS DE LA MORTALIDAD EN MÉxiCo}

DURANTE LAS Últimas dÉcadas, México ha expetimentado sensibles descensos en la mortalidad. Para el año 1940, cifras oficiales registraron una tasa bruta de mortalidad de 23.4 defunciones por cada 1000 habitantes y una esperanza de vida de 41.5 años; ${ }^{1}$ mientras que para el año 1978 las cifras fueron de 8.5 y 64 años respectivamente. ${ }^{2}$

En el abatimiento de los niveles de mortalidad y, consecuentemente, en las ganancias en la esperanza de vida, la trayectoria descendente de la mortalidad infantil ha jugado un papel muy importante. La mortalidad en el primer año de vida presentó en 1940 un nivel de 126 defunciones por cada 1000 nacidos vivos, ${ }^{3}$ mientras que la cifra correspondiente a 1978 se colocó en valores cercanos al 60 por mil. ${ }^{4}$ Aun cuando las deficiencias reconocidas en los registros de nacimientos y defunciones de menores de un año obligan a tomar estas cifras con precaución, ${ }^{5}$ es de aceptarse que la tasa de mortalidad infantil ha seguido cierta trayectoria descendente en los últimos 40 años.

A pesar de que ninguna ganancia en este sentido podría ser calificada como despreciable, no se puede estar satisfecho con el nivel de la tasa de mortalidad infantil alcanzado en México; sobre todo si se tiene en cuenta que, en la última

1 Dirección General de Estadística, SIC: Anuarios Estadísticos de los Estados Unidos Mexicanos (varios años).

2 Consejo Nacional de Población, México Demográfico, Breviario 1979.

3 Secretaría de Industria y Comercio, Dirección General de Estadística, Anuarios Estadísticos de los Estados Unidos Mexicanos.

4 Algunas de las clfras de mortalidad infantil en México en fechas recientes, que aparecen en dfversas publicaciones son las siguientes: $70.0 \%$ (1972-74), Secretaría de Programación y Presupuesto, Encuesta Mexicana de Fecundidad, Primer Informe Nacional 1979, p. 174; 66.0\% (1976-77) Population Reference Bureau. World Population Data Sheet: $56.8 \%$ (1979), Consejo Nacional de Población, México Demográfico, Breviario 1979.

5 Cordero, Eduardo "La Subestimación de la mortalidad infantil en México" Demografía y Economia, Vol, II, Núm. 1, 1968, pp. 44-62. 
década, varios países desarrollados $y$ en via de desarrollo han logrado triunfos mucho más marcados que México en la lucha contra la mortalidad infantil.

Para ilustrar el punto anterior basta como ejemplo observar los datos que aparecen a continuación sobre tasas de mortalidad infantil correspondiente a los años 1966 y 1976, en diferentes paises.

De acuerdo con el cuadro 1, países como Suecia, Noruega, Finlandia, y Dinamarca, aun partiendo de niveles bajos te mortalidad infantil en 1966, lograron diez años más tarde, reducciones entre el 28 y el $40 \%$. España, Francia, HongKong y Singapur partieron de cifras ligeramente más altas pero, en la misma década sus descensos están comprendidos entre el 43 y el $70 \%$. Por otro lado, países como Panamá, Costa Rica, Puerto Rico y Cuba muestran, en 1966, cifras menores que las mexicanas, sin embargo, durante el decenio que separa las dos observaciones, lograron descensos mucho mayores que el registrado en México.

\section{Cuadro 1}

TASAS DE MORTALIDAD INFANTIL 1966, 1976 EN ALGUNOS PAiSES DEL MUNDO Y DESCENSO PORCENTUAL OBTENIDO EN LA DÉCADA

\begin{tabular}{lrrr}
\hline & 1966 & 1976 & $\begin{array}{r}\text { Descenso porcentual ob- } \\
\text { tenido en la década }\end{array}$ \\
\hline Suecia & 12.6 & 8.3 & 34.1 \\
Noruega & 14.6 & 10.5 & 28.1 \\
Finiandia & 15.0 & 9.9 & 34.0 \\
Dinamarca & 16.9 & 10.2 & 39.6 \\
Francia & 21.7 & 10.4 & 52.1 \\
Hong-Kong & 23.9 & 13.7 & 42.7 \\
Singapur & 25.8 & 11.6 & 55.0 \\
España & 36.0 & 10.7 & 70.3 \\
Puerto Rico & 36.7 & $20.9 *$ & 43.1 \\
Cuba & 37.6 & 23.0 & 38.8 \\
Panamá & 45.0 & 35.6 & 20.9 \\
México & 62.9 & 54.7 & 13.0 \\
Costa Rica & 65.1 & 33.6 & 48.4 \\
\hline
\end{tabular}

NOTA: * Correspondiente a 1975

FUENTE: United Nations, De.mographic Yearbook 1970 y 1977.

\section{RELACIONES ENTRE MORT ALIDAD Y FACTORES SOCIOECONÓMICOS}

A principios de este siglo, en los países del mundo era notoria una relación inversa entre los niveles de desarrollo socioeconómico y la mortalidad general. A través del tiempo esta asociación se ha debilitado mucho, ya que el rápido descenso de la mortalidad en países en vías de desarrollo se ha debido, principalmente, a los avances teenológicos en la prevención y control de enfermedades y a la expansión de servicios médicos y de salud pública los cuales son, en gran medida, independientes de las condiciones económicas de la región. ${ }^{6}$ Aun cuando la afirmación

6 Naciones Unidas, Bolet in de Población Nümero 6, Naciones Unidas (1963), pp. 10 y 11. 
anterior es válida a nivel global existen evidencias de que dentro de cada país permanecen diferenciales en la mortalidad general por niveles socioeconómicos. ${ }^{7}$ En otras palabras, los estratos menos favorecidos de la población están en desventaja respecto al acceso y calidad de los servicios médicos y de la salud.

Así como la relación entre mortalidad general y desarrollo económico ha sido gradualmente menos significativa, es de esperarse que en el futuro suceda lo mismo que con la mortalidad infantil; sin embargo, en la actualidad existe consenso respecto de uma asociación entre mortalidad infantil y factores socioeconómicos.

Además de los factores económicos y sociales existe otro tipo de factores tales como sexo, orden de nacimiento, edad de la madre, intervalo intergenésico y otros, que también han sido vinculados con la mortalidad infantil. Con la inquietud de explorar, aunque sea sólo en parte, cuál es el peso que este segundo grupo de factores tiene en relación con los socioeconómicos, este estudio intenta valorar el impacto de algunas características asociadas con el niño y con el ambiente que le rodea, en el riesgo de alcanzar o no el primer año de vida.

Dado que en México un infante de cada 15. muere antes de completar su primer año de vida que, a pesar del desarrollo económico registrado en el país, el descenso en los niveles de mortalidad infantil ha sido leve, y que una de las principales preocupaciones del gobierno es la de preservar la vida de sus individuos, la identificación de los factores incidentes en la mortalidad infantil es fundamental para definir políticas y programas encaminados a prevenir, y consecuentemente, a reducir la mortalidad infantil.

\section{Fuente DE DATOS}

Este trabajo utiliza la información recolectada en la Encuesta Mexicana de Fe. cundidad que forma parte del programa denominado Encuesta Mundial de Fecun. didad. El proyecto fue financiado por el gobierno de México y por el Fondo de Naciones Unidas para Actividades de Población (UNFPA). El trabajo de campo fue llevado a cabo del 18 de julio de 1976 al 5 de marzo de 1977 por la Dirección General de Estadística con la cooperación dél Institu to de Investigaciones Sociales de la Universidad Nacional Autónoma de México (ISUNAM) y el asesoramiento del Centro de Estudios Económicos y Demográficos de El Colegio de México (CEED), el Consejo Nacional de Población (CONAPO) y el Sistema de Información para la Planificación Económica y Social (SIPES).

Esta encuesta fue la primera en su género a nivel nacional. Entre sus objetivos se contaron el obtener la información necesaria para analizar los niveles y patrones de fecundidad en el país así como incrementar el estudio científico de la fecundidad atendiendo a sus aspectos explicativos. ${ }^{8}$ Se efectuaron 13,200 entrevistas de hogar y de esa información se obtuvo una submuestra en la cual fueron elegibles, para ser entrevistadas individualmente, todas las mujeres de 20 a 49

7 A este respecto ver Aaron Antonovsky "Social Class Life Expectancy and Overal: Mortality" The Milbank-Memorial Found Quarterly, Vol. 45, Núms. 1-2, 1967.

8 Coordinación General del Sistema Nacional de Información Encuesta Mexicana de Fecundidad. Informe Metodológico, Secretaría de Programación y Presupuesto. México, octubre 1978, píg. 2. 
años de edad y aquellas de 15 a 19 años que estuvieran casadas o hubieran tenido algún hijo nacido vivo. Bajo estas condiciones se obtuvo información de 7,310 mujeres. Aun cuando esta encuesta no estuvo orientada hacia el análisis do la mortalidad, la información que contiene sobre la historia de embarazos y sobre las características socioeconómicas de las mujeres entrevistadas permite aproximarnos a nuestros objetivos.

La información obtenida en la Encuesta Mexicana de Fecundidad ha sido caracterizada como libre de inconsistencias y de errores de respuesta; por consiguiente puede confiarse en que los datos que se manejan en este estudio-evidencian la realidad del país. ${ }^{9}$

\section{METODOLOGIA}

La mortalidad infantil se define como el número de fallecimientos de niños menores de un año de edad, por mil nacidos vivos. Para propósitos de análisis, esta mortalidad es frecuentemente dividida entre mortalidad neonatal, que es aquélla que ocurre en el primer mes a partir del nacimiento, y mortalidad postneonatal, que es la que acontece durante el resto del primer año de vida. La diferencia entre mortalidad neonatal y postneonatal separa aproximadamente los factores endógenos y exógenos relacionados con la mortalidad infantil.

La mortalidad neonatal es asociada en gran medida, con factores genéticos tales como debilidad inherente de la madre o del feto, o bien con daños ocurridos durante la gestación o el nacimiento; en otras palabras, esta mortalidad está más o menos ligada a las causas de muerte difíciles de controlar en el nivel actua] del conocimiento médico. Por otra parte, se estima que la mortalidad postneonatal está conectada fundamentalmente con factores no biológicos incluyendo enfermedades, y por lo tanto, comprende aquellas causas de muerte que podrían haber sido prevenidas por la medicina moderna y campañas de salud pública. Aunque en no pocas defunciones infantiles es probable que causas endógenas y exógenas actúen conjuntamente, los grandes cambios acontecidos en los niveles de mortalidad infantil han sido debidos primordialmente a descensos en la mortalidad postneonatal.

En el análisis de los niveles, tendencias y diferenciales de la mortalidad infantil la medida más comúnmente usada es la tasa de mortalidad infantil. Esta tasa puede calcularse en forma directa dividiendo el número de fallecimientos de niños menores de un año de edad, entre el número total de nacidos vivos en el mismo período; también puede estimarse utilizando las funciones de una tabla de vida aplicando alguno de los métodos indirectos que, para derivarla, se basan en la proporción de hijos muertos según los grupos de edad de las mujeres. ${ }^{10} \mathrm{El}$ resultado en estos casos es una medida del fenómeno, a nivel agregado.

9 Respecto a la evaluación de la encuesta ver Ordorica y Potter, "An Evaluation of the Demographic Data Collected in the Mexican Fertility Survey" Draft for Circulation at the IUSSP Seminar on the Analysis of Maternity Histories, London, April 1980, The Population Council Working Paper. No. 4.

10 Por ejemplo: Brass, William, Seminario sobre métodos para medir variables demográficas (fecundidad y mortalidad), CELADE, San José, Costa Rica, 1973. Sullivan, Jeremian, 
Por otra parte, cuando se analizan datos a nivel individual, como es el caso que nos interesa, es frecuente medir la mortalidad infantil mediante la proporción de muertes infantiles que ha tenido cada mujer dentro del total de sus hijos nacidos vivos. Aunque ésta no es una medida inadecuada, tampoco es del todo satisfactoria. Como ejemplo basta considerar que si una mujer tuvo un hijo y se murió, tendrá la misma medida de mortalidad infantil que otra que, habiendo tenido cinco nacidos vivos, todos murieron. Aun cuando las proporciones son iguales, uno se inclinaría a especular que la mortalidad infantil en el segundo caso es más alta. Por otra parte, un cociente que envuelve sin distinción todas las muertes infantiles, como es el caso de la proporción antes mencionada, impide usar como variables explicativas, ciertas características individuales, como orden de nacimiento y edad de la madre al dar a luz, que pueden ser importantes en el estudio.

En la búsqueda de una mejor forma de abordar el microanálisis de la mortalidad infantil con una "representación" del fenómeno que pueda ser más sensible a los posibles determinantes, y permita identificar en forma más clara los factores que se asocian con la mortalidad infantil, decidimos examinar cada nacido vivo en forma individual. Es importante hacer hincapié en que, en este enfoque, la. unidad de análisis no es una proporción ni tampoco es una tasa; sino que es cada nacido vivo reportado en la encuesta. Dado que se carece de antecentes relacionados con el tipo de análisis propuesto, en este trabajo se intenta su aplicación y se somete a la consideración de los interesados en la materia, la validez del mismo.

Variable dependiente. Con el fin de obtener la variable dependiente en el análisis, todos los nacidos vivos son separados en dos grupos: por un lado los que murieron antes de alcanzar el primer año de vida, o sea las muertes infantiles; $y$ por otro, los que vivieron al menos hasta su primer aniversario. El primer grupo es a su vez separado en dos subgrupos: los que murieron durante las 4 primeras semanas de su existencia (mortalidad neonatal)y aquéllos que murieron entre el primero y el decimoprimer mes de vida (mortalidad postneonatal). Por falta de un nombre más apropiado a esta clasificación se la ha llamado "condición de su. pervivencia".

Conforme a la separación mencionada, a cada nacido vivo reportado en la encuesta le fue asignado el número 0,1 o 2 de acuerdo con sù condición de supervivencia en la forma siguiente:

(0) si murió durante el primer mes de vida (mortalidad neonatal)

(1) si murió entre el 10. y el 110. mes de vida (mortalidad postneonatal)

$(0)+(1)$ total de muertes infantiles

(2) si estaba vivo al momento de la encuesta y era mayor de 12 meses

\footnotetext{
"Models for the estimation of dying between birth and exact ages of early childhood" en Population Studies, marzo, 1972, págs. 79-97. Trussell, James, "A reestimation or the multiplying Factors for the Brass Technique for Determining Childhood Survivorship Rates", en Population Studies, marzo, 1975, pågs. 97-107. Feeney Griffith, "Estimación de tasas de mortalidad infantil a partir de información de sobrevivencia de hijos clasificados por edad de la madre", CELADE, Santiago de Chile, 1977.

Mina, V. Alejandro, "Estimaciones de los niveles, tendencias y diferenciales de la mortalidad infantil y en los primeros años de vida en México, 1940-1977, Demografía y Economia Vol. XV, Núm. 1 (45) El Colegio de México, 1981.
} 
de edad*, o bien, que vivió por lo menos hasta alcanzar un año đe vida (no importando si murió después).

Esta condición de supervivencia es precisamente nuestra variable dependiente.

Variable independiente. La relación entre mortalidad infantil y factores económicos, socioculturales y de cualquier otra indole, es bastante compleja. ni siquiera es fácil enumerar todos los factores que se mencionan como posibles determinantes: peso al nacer, sexo, raza, deficiencias nutricionales, acceso a servicios médicos, región de residencia, edad de la madre, orden de nacimiento, son sólo algunos de ellos. La labor se dificulta por la no disponibilidad de toda la información necesaria y porque a menudo las variables que se manejan están altamente correlacionadas entre sí.

Dado que la encuesta que se utiliza en este estudio no fue enfocada hacia la investigación de los determinantes de la mortalidad infantil, ha sido necesario restringir la exploración a la asociación entre la condición de supervivencia y las siguientes variables: edad de la madre en el momento del nacimiento, sexo del niño, orden de nacimiento, intervalo intergenésico, ${ }^{11}$ lactancia, previas pérdidas reproductivas, educación de la madre y residencia rural o urbana.

El procedimiento seguido en el estudio se puede resumir en los siguientes pasos:

10. De la historia de embarazos de las mujeres se seleccionó para su estudio la cohorte de nacimientos reportados en la encuesta como ocurridos entre 1971 y 1975.

2o. Mediante el uso de tablas cruzadas se realiza un análisis preliminar de las asociaciones entre la condición de supervivencia (porcentaje de 0,1 y 2 respecto al total de nacidos vivos encontrađos en cada columna es decir, en cada uno de los diferentes códigos, valores o características que tome la variable independiente), y las variables independientes.

3o. Se seleccionan las variables independientes que en los cruces mostraron cambios más pronunciados en la condición de supervivencia. En base a estos cambios se transforman en variables mudas.

40. Se determinan todas las posibles combinaciones de los valores de las variables independientes y mediante cruces simultáneos (multidimensionales) de ellos se encuentra el número total de nacidos vivos, separados conforme a su condición de superviviencia. El número de observaciones encontrado en cada caso se registra en la casilla respectiva.

5o. Con los resultados obtenidos en el punto anterior, se calculan las proporciones de supervivencia hasta un mes y hasta un año de vida.

6o. Con las proporciones de supervivencia como variable dependiente y los valores de las variables independientes (mudas) se efectúan los análisis de regresión múltiple. El número de casos está dado por el total de posibles combinaciones.

* Los niños vivos que al momento de la encuesta tenían entre 0 y 11 meses de edad, es decir todos los menores de un año, requerían una clasificación especial conforme a sus probabilidades de supervivencia hasta cumplir un año. Como esto quedaba al margen de la investigación planteada, fueron excluidos del estudio.

11 Intervalo intergenésico es el tiempo que media entre dos nacimientos sucesivos. Los interyalos entre el matrimonio y el nacimiento del primer nacido vivo (intervalo protogenési. co) no fueron incluidos en el análisis. 
Finalmente el estudio se enfoca en particular a la condición de supervivencia del último nacido vivo de cada mujer entrevistada. Esto permite que en la regresión, en lugar de las dicotomías, se utilicen los valores originales de las variables independientes.

\section{RESULTADOS}

El cuadro 2 presenta la distribución porcentual de la condición de supervivencia, tanto para el total de nacidos vivos entre los años 1940-1976, como para la cohorte de nacimientos ocurridos durante los cinco años anteriores al levantamiento de la encuesta. Puede observarse que las muertes infantiles representan, dentro del total de nacidos vivos, e] $8.9 \%$ o bien el 89 por mil, y en la cohorte $1972 \cdot 1976,85 \%$ o sea 85 por 1000 .

Estos índices de mortalidad infantil derivados de la condición de supervivencia presentan valores más altos que las tasas correspondientes a México 1966 y 1976 que parecen en el cuadro 1. Sin embargo, además de que los periodos de observación no son los mismos, es un hecho conocido que en el país existe una subestimación de este indicador. ${ }^{12}$ Por otra parte, los resultados son bastante cercanos a la cifra de 85.7 muertes por cada mil nacimientos obtenida al aplicar a la misma información, el método de Jorge L. Somoza. ${ }^{13}$

También en el cuadro 2 puede observarse que los índices de mortalidad neonatal son superiores a los de mortalidad postneonatal. Esto está relacionado con el hecho de que el $51 \%$ del total de muertes infantiles ocurre en el primer mes de vida y el $49 \%$ restante queda distribuido durante los 11 meses siguientes (véase en la gráfica 1 la distribución porcentual del total de muertes infantiles de acuerdo con la edad del niño en meses cumplidos al morir).

A continuación se presentan los resultados de los cruces de la condición de supervivencia con cada una de las variables independientes.

\section{SEXO DEL NIÑ̃o}

En la mayoria de los países se observa que por cada 100 mujeres nacen aproximadamente 105 hombres, y también que la mortalidad masculina es, a lo largo de todas las edades, superior a la femenina. En la encuesta mexicana por lo general, estas dos relaciones se cumplen. Por un lado en el número reportado de nacimientos la diferencia por sexo es más marcada en la mortalidad neonatal que en la postneonatal.

12 Eduardo Cordero, Evaluación de la mortalidad infantil en la República Mexicana 1930-1970, prólogo a "Evaluación y Análisis", Serie 1ü, Núm. 1, 1975. También Ordorica y Potter, op. cit., pág. 28.

13 Mina, Alejandro op. cit., pág. 89. El método aplicado se encuentra en Jorge L. Somo. za, "Estimaciones de la mortalidad al comienzo de la vida en Colombia basadas en información de la encuesta nacional de fecundidad 1976". CELADE. Santiago de Chile, 1979. 
Cuadro 2

MÉXICO: ALGUNOS FACTORES ASOCIADOS CON LA MORTALIDAD INFANTIL, DISTRIBUCIÓN PORCENTUAL DE LA CONDICIÓN DE SOBREVIVIENTES PARA EL TOTAL DE NACIDOS VIVOS PARA LA COHORTE 1970-1975

\begin{tabular}{lcccc}
\hline Condición de supervivencia & $\begin{array}{c}\text { Clave de } \\
\text { identificación }\end{array}$ & $\begin{array}{c}\text { Total } \\
1940-1976\end{array}$ & $\begin{array}{c}\text { Cohorte } \\
1970-1975\end{array}$ \\
\hline Muertes infantiles & $(0+1)$ & $\%$ & 8.6 & 6.88 \\
Mortalidad neonatal & & $\mathrm{N}$ & $(2311)$ & $(532)$ \\
(No llegaron a cumplir un mes de vida, & $(0)$ & $\%$ & 4.4 & 3.5 \\
murieron durante el primer mes) & & $\mathbf{N}$ & $(1174)$ & $(274)$ \\
$\begin{array}{l}\text { Mortalidad postneonatal } \\
\text { (No llegaron a cumplir un año de vida, }\end{array}$ & $(1)$ & $\%$ & 4.2 & 3.3 \\
murieron entre 1 y 11 meses) & & $\mathrm{N}$ & $(1137)$ & $(258)$ \\
Sobrevivientes hasta el primer año de vida, & $(2)$ & $\%$ & 91.4 & 93.2 \\
Total de nacidos vivos considerados & $(0+1+2)$ & $\%$ & $(24567)$ & $(7265)$ \\
& & $\mathrm{N}$ & $(26878)$ & 100.0 \\
& & & & $(7797)$
\end{tabular}

\footnotetext{
$\%$ Porcentaje respecto al total considerado en cada periodo N Número de casos.

FUENTE: Encuesta Mexicana de Fecundidad 1976.
} 


\section{Gráfica 1}

MÉXICO: DISTRIBUCIÓN RELATTVA DEL NÚMERO TOTAL DE DEFUNCIONES INFANTILES POR EDAD (MESES SOBREVIVIDOS A LA MUERTE), COHORTE DE NACIMIENTOS 1970-1975

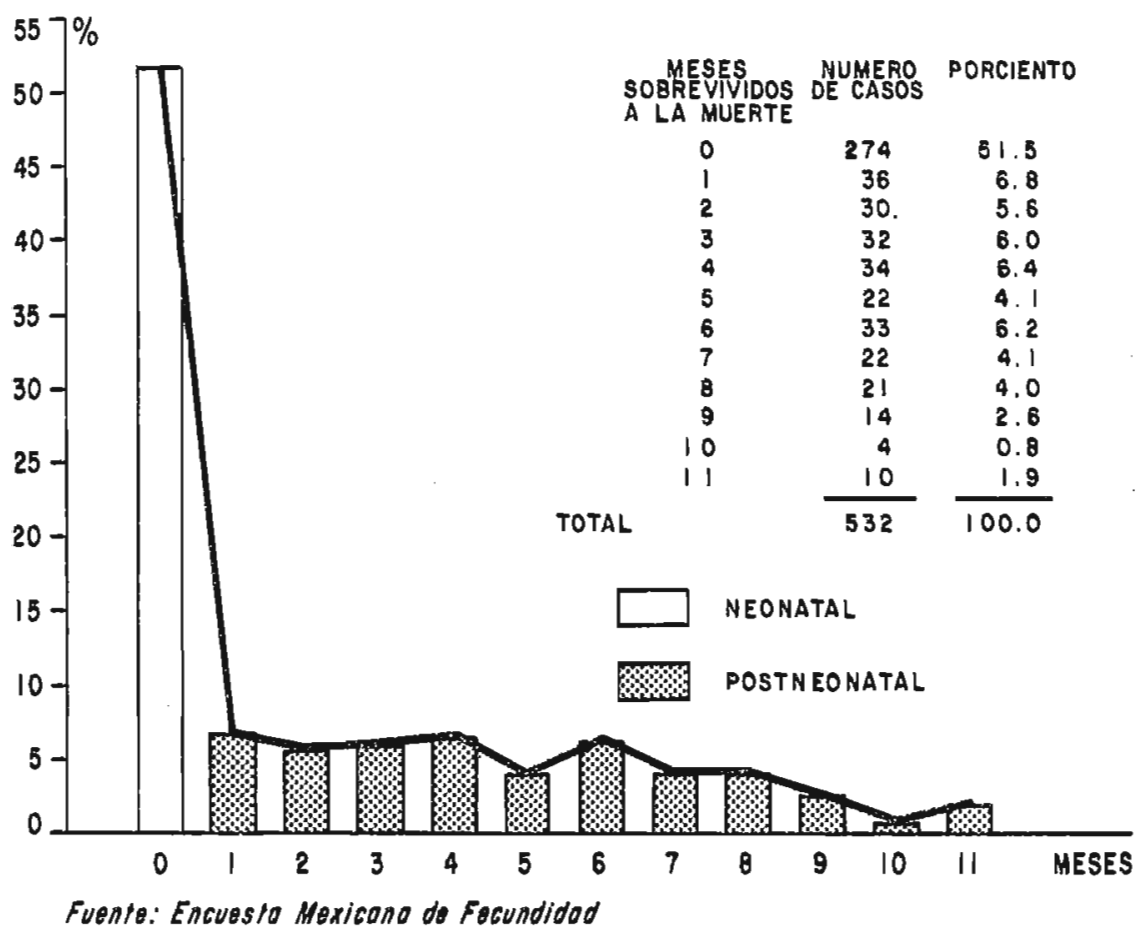

ORDEN DE NACIMIENTO

Varios estudios entre ellos el de Puffer y Serrano, encontraron que el orden de nacimiento es un importante determinante de la mortalidad infantil. ${ }^{14}$ Por lo general infantes de alto orden de nacimiento tienen menos probabilidades de vivir que aquéllos de los primeros órdenes. El cuadro 4 registra la condición de supervivencia por orden de nacimiento; como en el caso anterior se presentan los resultados tanto para el total de nacimientos como para la cohorte 1970-1975.

Aun cuando los resultados deben tomarse con precaución porque en ocasiones el número de casos en cada celda es muy pequeño, de la observación de los totales de muertes infantiles, así como de los de mortalidad neonatal y postneonatal podría inferirse que en base a los datos mexicanos, la asociación entre orden de nacimiento y mortalidad infantil, si acaso existe, sólo se da en órdenes superiores al quinto.

14 Puffer, R. y Serrano, C. El peso al nacer, la edad materna y el orden de nacimiento: importantes determinantes de la mortalidad infantil. UPS, Publicación científica No. 294. 1975. 
Cuadro 3

MÉXICO: ALGUNOS FACTORES ASOCIADOS CON LA MORTALIDAD INFANTIL CONDICIÓN DE SOBREVIVENCIA POR SEXO

\begin{tabular}{lccccr}
\hline $\begin{array}{l}\text { Condición de } \\
\text { supervivencia }\end{array}$ & $\begin{array}{c}\text { Clave de } \\
\text { identificación }\end{array}$ & & \multicolumn{2}{c}{$\begin{array}{c}\text { Cohorte de nacimientos 1970-1975 } \\
\text { hombres }\end{array}$} & $\begin{array}{r}\text { mujeres } \\
\text { total }\end{array}$ \\
\hline Defunciones infantiles & $(0+1)$ & $\%$ & 7.9 & 5.7 & 6.8 \\
Mortalidad neonatal & & $N$ & $(312)$ & $(220)$ & $(532)$ \\
& $(0)$ & $\%$ & 4.1 & 2.9 & 3.5 \\
Mortalidad postneonatal & $(1)$ & $\mathrm{N}$ & $(163)$ & $(111)$ & $(274)$ \\
& & $\%$ & 3.8 & 2.8 & 3.3 \\
Sobrevivientes hasta el primer & $(2)$ & $\%$ & $(149)$ & $(109)$ & $(258)$ \\
año de vida & & $\mathrm{N}$ & $(3654)$ & $(3611)$ & $(7265)$ \\
Total de nacidos vivos & total & $\%$ & 100.0 & 100.0 & 100.0 \\
considerados & & $\mathrm{N}$ & $(3966)$ & $(3831)$ & $(7797)$ \\
\hline
\end{tabular}

\% Distribución porcentual.

N Número de casos.

FUENTE: Encuesta Mexicana de Fecundidad 1976. 
Cuadro 4

MÉXICo: ALgunOS FACTORES ASOCIADOS CON LA MORTALIDAD INFANTIL, CONDICIÓN DE SUPERVIVENCIA POR ORDEN DE NACIMIENTO

\begin{tabular}{|c|c|c|c|c|c|c|c|c|c|}
\hline & \multirow{2}{*}{\multicolumn{2}{|c|}{$\begin{array}{c}\text { Clave de } \\
\text { identificación }\end{array}$}} & \multicolumn{7}{|c|}{$\begin{array}{l}\text { Cohorte de nacimientos } 1970-1975 \\
\text { Orden de nacimientos }\end{array}$} \\
\hline & & & $1^{0}$ & $2^{\circ}, 3^{\circ}$ & $4^{\circ}, 5^{\circ}$ & $6^{\circ}, 7^{\circ}$ & $8^{\circ}, 90$ & $10^{\circ} y+$ & Total \\
\hline Defunciones infantiles & $(0+1)$ & $\begin{array}{l}\% \\
\mathrm{~N}\end{array}$ & $\begin{array}{l}6.8 \\
(97)\end{array}$ & $\begin{array}{c}6.0 \\
(133)\end{array}$ & $\begin{array}{c}6.3 \\
(100)\end{array}$ & $\begin{array}{l}7.2 \\
(83)\end{array}$ & $\begin{array}{l}6.1 \\
(47)\end{array}$ & $\begin{array}{c}11.4 \\
(72)\end{array}$ & $\begin{array}{c}6.8 \\
(533)\end{array}$ \\
\hline Mortalidad neonatal & (0) & $\stackrel{\%}{N}$ & $\begin{array}{l}3.7 \\
(53)\end{array}$ & $\begin{array}{l}2.7 \\
(60)\end{array}$ & 3.2 & $\begin{array}{l}4.0 \\
(46)\end{array}$ & $\begin{array}{l}3.1 \\
(24)\end{array}$ & $\begin{array}{l}6.3 \\
(40)\end{array}$ & $\begin{array}{c}3.5 \\
(274)\end{array}$ \\
\hline Mortalidad postneonatal & (1) & $\stackrel{\%}{N}$ & $\begin{array}{l}3.1 \\
(44)\end{array}$ & $\begin{array}{l}3.3 \\
(73)\end{array}$ & $\begin{array}{l}3.1 \\
(49)\end{array}$ & $\begin{array}{l}3.2 \\
(37)\end{array}$ & $\begin{array}{l}3.0 \\
(23)\end{array}$ & $\begin{array}{l}5.1 \\
(32)\end{array}$ & $\begin{array}{c}3.3 \\
(258)\end{array}$ \\
\hline $\begin{array}{l}\text { Sobrevivientes hasta } \\
\text { el primer año de vida }\end{array}$ & (2) & $\stackrel{\%}{N}$ & $\begin{array}{c}93.2 \\
(1327)\end{array}$ & $\begin{array}{l}94.0 \\
(2102)\end{array}$ & $\begin{array}{c}93.7 \\
(1489)\end{array}$ & $\begin{array}{c}92.8 \\
(1067)\end{array}$ & $\begin{array}{l}92.9 \\
(720)\end{array}$ & $\begin{array}{l}88.6 \\
(560)\end{array}$ & $\begin{array}{c}93.2 \\
(7265)\end{array}$ \\
\hline Totales & & $\frac{\%}{N}$ & $\begin{array}{l}100.0 \\
(1424)\end{array}$ & $\begin{array}{l}100.0 \\
(2235)\end{array}$ & $\begin{array}{l}100.0 \\
(1589)\end{array}$ & $\begin{array}{l}100.0 \\
(1150)\end{array}$ & $\begin{array}{c}100.0 \\
(767)\end{array}$ & $\begin{array}{r}100.0 \\
(632)\end{array}$ & $\begin{array}{l}100.0 \\
(7797)\end{array}$ \\
\hline
\end{tabular}

$\%$ Porcentaje con respecto a la columna.

N Número de casos.

FUENTE: Encuesta Moxicana de Fecundidad 1976. 
Cuadro 5

MÉXICO: ALGUNOS FACTORES ASOCLADOS CON LA MORTALWAD INFANTL, CONDICIÓN DE SUPERVIVENCIA POR INTERVALO INTERGENÉSICO

\begin{tabular}{|c|c|c|c|c|c|c|c|c|c|c|}
\hline & \multirow{3}{*}{$\begin{array}{c}\begin{array}{c}\text { Clave de } \\
\text { identificación }\end{array} \\
(0+1)\end{array}$} & & \multicolumn{5}{|c|}{$\begin{array}{l}\text { Cohorte de nacimientos } 1970-1975 \\
\text { Intervalo intergenésico }\end{array}$} & \multirow{2}{*}{$\begin{array}{r}\text { len } n \\
37-48\end{array}$} & \multicolumn{2}{|c|}{ meses) } \\
\hline & & & $\begin{array}{c}\text { Menos } \\
\text { de } 8 \\
\text { os exclu }\end{array}$ & $\operatorname{dos}^{8-11}$ & $12-18$ & $19-24$ & $25-36$ & & $49 y+$ & Total \\
\hline Defunciones infantiles & & $\stackrel{\%}{N}$ & $\begin{array}{c}7.8 \\
(118)\end{array}$ & $\begin{array}{l}14.1 \\
(42)\end{array}$ & $\begin{array}{c}8.2 \\
(122)\end{array}$ & $\begin{array}{l}5.5 \\
(81)\end{array}$ & $\begin{array}{c}5.7 \\
(100)\end{array}$ & $\begin{array}{l}5.5 \\
(36)\end{array}$ & $\begin{array}{l}5.3 \\
(33)\end{array}$ & $\begin{array}{r}6.6 \\
(414)\end{array}$ \\
\hline Mortalidad neonatal & (0) & $\stackrel{0}{\mathbf{g}}$ & $\begin{array}{l}4.4 \\
(67)\end{array}$ & $\begin{array}{l}8.1 \\
(24)\end{array}$ & $\begin{array}{l}3.9 \\
(58)\end{array}$ & 2.5 & 3.1 & $\begin{array}{l}2.9 \\
(19)\end{array}$ & $\begin{array}{l}2.4 \\
(15)\end{array}$ & $\begin{array}{r}3.3 \\
(207)\end{array}$ \\
\hline Mortalidad postneonatal & (1) & $\%$ & $\begin{array}{l}3.4 \\
(51)\end{array}$ & $\begin{array}{l}6.0 \\
(18)\end{array}$ & $\begin{array}{l}4.3 \\
(64)\end{array}$ & $\begin{array}{l}3.0 \\
(44)\end{array}$ & $\begin{array}{l}2.6 \\
(46)\end{array}$ & $\begin{array}{l}2.6 \\
(17)\end{array}$ & $\begin{array}{l}29 \\
(18)\end{array}$ & $\begin{array}{r}3.3 \\
(207)\end{array}$ \\
\hline $\begin{array}{l}\text { Sobrevivientes hasta } \\
\text { el primer año de vida }\end{array}$ & (2) & $\frac{0}{6}$ & $\begin{array}{c}92.2 \\
(1389)\end{array}$ & $\begin{array}{l}85.9 \\
(255)\end{array}$ & $\begin{array}{c}91.8 \\
(1374)\end{array}$ & $\begin{array}{c}94.5 \\
(1390)\end{array}$ & $\begin{array}{c}94.3 \\
(1643)\end{array}$ & $\begin{array}{l}94.5 \\
(621)\end{array}$ & $\begin{array}{l}94.7 \\
(593)\end{array}$ & $\begin{array}{r}93.4 \\
(5876)\end{array}$ \\
\hline Totales & & $\stackrel{\%}{\mathrm{~N}}$ & $\begin{array}{l}100.0 \\
(1507)\end{array}$ & $\begin{array}{c}100.0 \\
(297)\end{array}$ & $\begin{array}{l}100.0 \\
(1496)\end{array}$ & $\begin{array}{l}100.0 \\
(1471)\end{array}$ & $\begin{array}{l}100.0 \\
(1743)\end{array}$ & $\begin{array}{c}100.0 \\
(657)\end{array}$ & $\begin{array}{c}100.0 \\
(626)\end{array}$ & $\begin{array}{r}100.0 \\
(6290)\end{array}$ \\
\hline
\end{tabular}

Casos eliminados 1 507. Nacimientos prematuros o nacimientos múltiples.

\% Porcentaje respecto al total de cada columna.

N Número de casos. 
Sin embargo, cuando conjuntamente se observan las variaciones en la condición de supervivencia por orden de nacimiento y por edad de la madre al dar a luz, dos importantes hechos se manifiestan:

1. Cuando tanto el primero como el segundo hijo se tienen después de los 20 años, el riesgo de muerte infantil es menor que si se tienen antes de los 20 años.

2. En órdenes superiores de nacimiento, la mortalidad infantil es más alta cuando la madre es joven, que cuando la madre tiene una edad más avanzada. Este hecho desde luego está relacionado con la frecuencia con que se tienen los niños y consecuentemente, con los intervalos entre nacimientos.

\section{Intervalo entre el nacimiento observado y el anterior}

Un corto intervalo entre dos embarazos, y por tanto una rápida sucesión de nacimientos, estará asociado con menor tiempo de lactancia (en el caso de que la haya) y una probable división de los cuidados de la madre entre dos o más infantes. Cuando un infante es alimentado con leche materna y ésta debe ser suprimida por la llegada de otro nifio, el cambio hacia leche artificial, tal vez escasa en proteínas o contaminada por microbios, deja al infante vulnerable a infecciones. En el segundo caso, los niños pequeños están expuestos a diversas clases de accidentes la mayoría de los cuales se pueden evitar mediante una atención adecuada.

La condición de supervivencia por intervalo intergenésico aparece en el cuadro $5 .^{15}$ Un hecho que salta a la vista es que los más altos riesgos de muerte infantil se encuentran cuando el intervalo que media entre dos nacimientos sucesivos es menor que un año. Le sigue en importancia, pero con un riesgo de muerte considerablemente más bajo, el intervalo entre 12 y 18 meses. A partir de entonces y hasta llegar a los 48 meses, por lo general a medida que el intervalo aumenta, el riesgo disminuye.

Aparentemente la relación inversa entre intervalo intergenésico y mortalidad infantil se modifica y cambia de dirección cuando el tiempo transcurrido entre dos nacidos vivos es superior a los 4 años. Aunque es de aceptarse que los intervalos muy largos estén asociados con una mayor mortalidad infantil, la disminución del número de acontecimientos en las celdas obliga a tomar las cifras con precaución. Además, pone de manifiesto la poca frecuencia con que se dan esos casos y por lo mismo la poca importacia que representan dentro del contexto mexicano.

\section{Existencia de previas pérdidas reproductivas}

MacMahon, en su estudio sobre mortalidad infantil en los Estados Unidos observó que las madres que habian tenido algún embarazo anterior que terminó en

15 Es pertinente mencionar que, debido a que los nacimientos de primer orden son ex. cluidos cuando se maneja la variable intervalo entre dos nacimientos sucesivos, el número de nacidos vivos que aparece en estas tabulaciones es inferior al reportado en las tablas donde se presentan las otras variables independientes. 
muerte fetal tendían a tener otra pérdida. ${ }^{16}$ Por otro lado, Shapiro encontró que en las mujeres cuyo último embarazo habis terminado en muerte fetal o muerte infantil tenian cierta tendencia a' que la anomalia se repitiera. ${ }^{17}$ En nuestro estudio hemos considerado para cada caso, las muertes fetales anteriores (abortos y nacidos muertos), así como también la mortalidad infantil anterior, con el objeto de observar su influencia en la mortalidad infantil. En forma similar a lo encontrado en Estados Unidos, los datos mexicanos muestran una asociación entre la existencia de previas pérdidas y la mortalidad infantil.

En el cuadro 6 se puede observar que, los menores indices de mortalidad infantil se registran cuando la madre no ha sufrido con anterioridad pérdidas reproductivas. Por otro lado, si la mujer tiene en su pasado muertes infantiles o embarazos terminados en aborto o nacido muerto, las probabilidades de padecer muerte infantil son mayores.

Las cifras de la tabla muestran que a medida que aumenta el número de previas pérdidas reproductivas aumenta también la mortalidad infantil. Los indices de muertes neonatales de las mujeres que han tenido tres o más pérdidas comparados con los de aquéllas que no han tenido ninguna pérdida en más del doble y si el análisis se continúa hasta cuatro y $\mathrm{u}$ más perdidas los índices casi se llegan a cuadruplicar.

\section{Lactancia}

La leche materna provee todo lo que es requerido para una adecuada alimentación en los primeros seis meses de vida y, desde luego, contiene mayor valor nutritivo que las fórmulas o comidas sustitutas. La lactancia contribuye también a espaciar los nacimientos porque, en un considerable número de casos, prolonga el tiempo de amenorrea después del parto, $\mathrm{y}$ con esto, ofrece protección para un nuevo embarazo. ${ }^{18}$ Por otra parte, la lactancia evita el riesgo de infecciones derivadas de la falta de higiene con la preparación de los biberones y demás utensilios usados en la lactancia artificial.

Uno de los principales hallazgos de la Encuesta de la Organización Panamericana de la Salud en la que se relaciona Ja mortalidad con diversos factores, fue que la desnutrición juega un papel central como causa directa de la mortalidad en los primeros años de vida. ${ }^{19}$ Por otro lado, en los Anuarios Estadísticos de los

16 MacMahon, Brian, Mary Grace Kovar, and Jacob J. Feldman, "Infant mortality rates: Socioeconomic factors (Washington, D. C.): Department of Health, Education, and Welfare Publication No. (HSM) 72-1045, 1972.

17 Shapiro, S., I. J. Ross and H. S. Levine, "Relationship of selected prenatal factors to pregriancy outcome and congenital anomalies", American Journal of Public Healt, Vol. 55 (1965), pp. 268-282.

$18 \mathrm{H}$. Leridon, Human fertility: the basic components, The University of Chicago Press, 1977, pág. 83.

19 Puffer, Ruth Rice y Carlos V. Serrano, Patterns of mortality in childhood: Report of the Inter-American Investigation of Mortality in Childhood, Washington, D. C.: Pan American Health Orgunization, (1973) págs. 345-355. Esta publicición presenta los resultados de un estudio de 5 años que concluyó en 1972 sobre mortalidad infantil en países subdesarrollados. 


\section{Cuadro 6}

MÉXICO: ALGUNOS FACTORES ASOCIADOS CON LA MORTALIDAD INFANTIL, CONDICIÓN DE SUPERVIVENCIA POR NÚMERO DE PREVIAS PÉRDIDAS REPRODUCTIVAS

\begin{tabular}{|c|c|c|c|c|c|c|c|}
\hline \multirow[b]{3}{*}{ Defunciones infantiles } & \multirow{3}{*}{$\begin{array}{c}\begin{array}{c}\text { Clave de } \\
\text { identificación }\end{array} \\
(0+1)\end{array}$} & \multirow{4}{*}{$\begin{array}{l}\% \\
\mathbf{N} \\
\% \\
\mathbf{0} \\
\mathbf{N}\end{array}$} & \multicolumn{5}{|c|}{$\begin{array}{l}\text { Cohorte de nacimientos } 1970-1975 \\
\text { Número de pérdidas reproductivas }\end{array}$} \\
\hline & & & 0 & 1 & 2 & $3 y+$ & Total \\
\hline & & & $\begin{array}{c}5.9 \\
(296)\end{array}$ & $\begin{array}{c}7.7 \\
(120)\end{array}$ & $\begin{array}{l}7.8 \\
(49)\end{array}$ & $\begin{array}{l}11.2 \\
(67)\end{array}$ & $\begin{array}{c}6.8 \\
(532)\end{array}$ \\
\hline Mortalidad neonatal & (0) & & $\begin{array}{c}2.8 \\
(142)\end{array}$ & $\begin{array}{l}4.1 \\
(64)\end{array}$ & $\begin{array}{l}4.3 \\
(27)\end{array}$ & $\begin{array}{l}6.9 \\
(41)\end{array}$ & $\begin{array}{c}3.5 \\
(274)\end{array}$ \\
\hline Mortalidad postneonatal & (1) & $\begin{array}{l}\% \\
\mathbf{N}\end{array}$ & $\begin{array}{c}3.1 \\
(154)\end{array}$ & $\begin{array}{l}3.6 \\
(56)\end{array}$ & $\begin{array}{l}3.5 \\
(22)\end{array}$ & $\begin{array}{l}4.3 \\
(26)\end{array}$ & $\begin{array}{c}3.3 \\
(258)\end{array}$ \\
\hline $\begin{array}{l}\text { Sobrevivientes hasta el } \\
\text { primer año de vida }\end{array}$ & (2) & $\begin{array}{l}\% \\
\mathbf{N}\end{array}$ & $\begin{array}{c}94.1 \\
(4704)\end{array}$ & $\begin{array}{c}92.3 \\
(1447)\end{array}$ & $\begin{array}{l}92.2 \\
(582)\end{array}$ & $\begin{array}{l}88.8 \\
(532)\end{array}$ & $\begin{array}{c}93.2 \\
(7265)\end{array}$ \\
\hline $\begin{array}{l}\text { Total de nacidos vivos } \\
\text { considerados }\end{array}$ & Total & $\begin{array}{l}\% \\
\mathbf{N}\end{array}$ & $\begin{array}{l}100.0 \\
(5000)\end{array}$ & $\begin{array}{l}100.0 \\
(1567)\end{array}$ & $\begin{array}{r}100.0 \\
(631)\end{array}$ & $\begin{array}{c}100.0 \\
(599)\end{array}$ & $\begin{array}{l}100.0 \\
(7797)\end{array}$ \\
\hline
\end{tabular}

Distribución porcentual.

N Número de casos.

FUENTE: Encuesta Mexicana de Fecundidad 1976. 


\section{Cuadro 7}

MÉXICO: ALGUNOS FACTORES ASOCIADOS CON LA MORTALIDAD INFANTIL, CONDICIONES DE SUPERVIVENCIA POR LACTANCIA

\begin{tabular}{|c|c|c|c|c|c|c|}
\hline & \multirow[t]{2}{*}{$\begin{array}{c}\text { Clave de } \\
\text { identificación }\end{array}$} & \multicolumn{5}{|c|}{$\begin{array}{c}\text { Cohorte de nacimiento } 1970-1975 \\
\text { Lactancia }\end{array}$} \\
\hline & & & $\begin{array}{l}\text { cluidos } \\
\text { Resp. }\end{array}$ & $S I$ & NO & Total \\
\hline Defunciones infantiles & $(0+1)$ & $\stackrel{\%}{N}$ & $\begin{array}{l}19.3 \\
(11)\end{array}$ & $\begin{array}{c}2.9 \\
(180)\end{array}$ & $\begin{array}{l}21.7 \\
(341)\end{array}$ & $\begin{array}{c}6.7 \\
(521)\end{array}$ \\
\hline Mortalidad neonatal & $(0)$ & 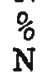 & $\begin{array}{r}0.00 \\
(0)\end{array}$ & $\begin{array}{l}0.1 \\
(9)\end{array}$ & $\begin{array}{l}16.9 \\
(265)\end{array}$ & $\begin{array}{c}3.5 \\
(274)\end{array}$ \\
\hline Mortalidad postneonatal & (1) & $\stackrel{0}{N}$ & $\begin{array}{l}19.3 \\
(11)\end{array}$ & $\begin{array}{c}2.8 \\
(171)\end{array}$ & $\begin{array}{l}4.8 \\
(76)\end{array}$ & $\begin{array}{l}3.2 \\
(247)\end{array}$ \\
\hline $\begin{array}{l}\text { Sobrevivientes hasta el } \\
\text { primer año de vida } \\
\text { Tot a les }\end{array}$ & (2) & $\begin{array}{l}\% \\
\mathrm{~N} \\
\% \\
\mathrm{~N}\end{array}$ & $\begin{array}{r}80.7 \\
(46) \\
100.0 \\
(57)\end{array}$ & $\begin{array}{l}97.1 \\
(5990) \\
100.0 \\
(6170)\end{array}$ & $\begin{array}{l}78.3 \\
(1227) \\
100.0 \\
(1568)\end{array}$ & $\begin{array}{l}93.3 \\
(7217) \\
100.0 \\
(7738)\end{array}$ \\
\hline
\end{tabular}

No respuesta 57.

Casos eliminados.

\% Distribución porcentual.

N Número de casos.

FUENTE: Encuesta Mexicana de Fecundidad 1976.

Estados Unidos Mexicanos las enfermedades infecciosas y parasitarias aparecen siempre entre los dos primeros lugares dentro de las causas de muerte infantiles. ${ }^{20}$ Dado que tanto la desnutrición como las infecciones intestinales se encuentran enre las principales causas de muerte, $y$ en gran medida ambas pueden ser evitadas mediante la alimentación materna, es de esperarse que la lactancia y el tiempo que se recibe (al menos hasta los 6 meses) estén asociados con una menor mortalidad infantil.

En la Encuesta Mexicana de Fecundidad se preguntó a la entrevistada si le había dado èl pecho y por cuánto tiempo, al último y al penúltimo de sus hijos. Conforme a los resultados presentados en el Primer Informe Nacional de la En. cuesta Mexicana de Fecundidad, $85 \%$ de las mujeres alguna vez unidad con al menos 2 nacidos vivos respondió afirmativamente. El promedio reportado de tiempo de lactancia es 9.7 meses y la moda, es decir, la mayor frecuencia observada, es de 12 meses.

De lo anterior puede inferirse que en los primeros meses de vida de los niños mexicanos, recibir alimento materno es más la regla que la excepción. Vale la pena hacer notar que en las tabulaciones del mencionado informe también se observa una relación inversa entre tiempo de lactancia y escolaridad de la madre, así como entre tiempo de lactancia y tamaño de localidad de residencia. ${ }^{21}$ En

20 Dirección General de Estadística, S.I.C. Anuarios Estadísticos (Varios años).

21 Secretaría de Programación y Presupuesto, Encuesta Mexicana de Fecundidad, Ier. Informe Nacional. Vol. II, pp. 447-455. 
vista de que el proceso de desarrollo está asociado con aumentos en los niveles de escolaridad y de urbanización, es de esperarse que, si la relación irversa persiste, a medida que el país se modernice se registre una disminución en el tiempo promedio de lactancia.

Como quedó dicho anteriormente, no se cuenta con información sobre lactancia para cada nacido vivo. Bajo la hipótesis de que la respuesta a la pregunta, que sobre el particular fue hecha para el último y penúltimo hijo, es asimismo válida para los hijos anteriores que la mujer dio a luz, se atribuyó a cada nacido vivo la existencia o falta de lactancia. Somos conscientes de que este supuesto en algunos casos puede no cumplirse, pero creemos que en la mayoría de los casos es verdadero. Más aún, dado que sólo estamos incluyendo nacimientos ocurridos en un período de cinco años, el número de mujeres que tuvieron más de dos hijos en ese tiempo debe ser bajo.

El cuadro 7 muestra que, si el supuesto anterior es válido del total de nacidos vivos considerados en el estudio, la gran mayoría (casi $89 \%$ ) recibió leche materna. Respecto a la influencia que tiene la lactancia en la supervivencia infantil se observa que los porcentajes de mortalidad neonatal, postneonatal $y$, consecuentemente, mortalidad infantil, son más altos para los niños que no fueron amamantados que para aquéllos que sí lo fueron. Esto es consistente con los hallazgos de Alan Berg cuando reporta que la falta de leche materna en el nifio es una de las mayores causas de malnutrición y mortalidad. ${ }^{22}$

\section{Residencia rural-urbana}

Es conocido que las diferencias rural-urbanas en los niveles de mortalidad infantil son, en algunos casos, resultado de diferencias socioeconómicas y que a medida que los países avanzan en el proceso de desarrollo, las diferenciales tienden a disminuir. Datos recientes muestran que en los países industrializados los niveles de mortalidad infantil en las zonas urbanas, comparados con los niveles de la mortalidad infantil de las rurales, no siguen un patrón definido: algunos registran sobremortalidad rural, otros sobremortalidad urbana y otros más presentan cifras similares para ambas áreas. Lo que sí es notorio es que cuando los valores no son uniformes, las diferencias existentes son de poca magnitud. ${ }^{23}$

Por otra parte, en los países poco industrializados los niveles de mortalidad infantil de las zonas urbanas son más bajos que los de las zonas rurales. La ventaja urbana está asociado, entre otros factores, a la mejor infraestructura y disponibilidad de los servicios médicos, hospitalarios y de salud pública, a los más altos niveles educativos, al mayor ingreso y a las mejores condiciones sanitarias.

El criterio por el cual se distinguen las localidades urbanas de las rurales varía entre países y también a través del tiempo.

En el análisis de la encuesta hemos escogido el limite de 20000 habitantes para

22 Berg, Alan, The nutrition factor: its role in national development The Brookings Institution, Washington, D. C.

23 Naciones Unidas, Factores Determinantes y Consecuencias de las Tendencias Demográficas, Volumen 1, Naciones Unidas ST/SUA/SER.A/50, pp. 140-142. También Demographic Yearbook Varios años. 


\section{Grática 2}

MÉXICO: MORTALIDAD INFANTIL, PORCENTAJE DE MUERTES INFANTILES NEONATALES Y POSTNEONATALES CORRESPONDIENTES A ZONAS

RURALES Y URBANAS, COHORTE DE NACIMIENTOS 1970-1975

$\%\left[\begin{array}{l}80 \\ -70 \\ -60 \\ -50 \\ -40 \\ -30 \\ -20 \\ -10 \\ 0\end{array}\right.$

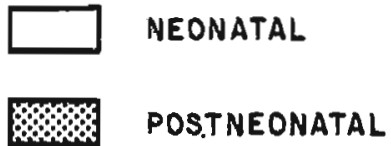

POSTNEONATAL

diferenciar lo urbano de 10 rural. ${ }^{24}$ Con este criterio, del total de mujeres entrevistadas $48 \%$ residía en zonas urbanas y $52 \%$ en localidades con menos de 20000 habitantes. Respecto al total de nacidos vivos considerados en el estudio, los porcentajes son 44 y 56 para localidades urbanas y rurales respectivamente. Estas cifras están relacionadas con el hecho de que en las zonas ruraleś de México se registra una fecundidad más alta que en las zonas urbanas.

El cuadro 8 presenta, tanto para las zonas rurales como para las urbanas, la conđición de supervivencia y, la gráfica 2 los porcentajes de defunciones infantiles, neonatal y postneonatal, según residencia rural o urbana.

Aun cuando podría argumentarse la posibilidad de que, debido a la migración, algunos de los nacimientos y muertes de los hijos de las entrevistadas hayan ocurrido en localidades con tamaño diferente del de la localidad donde fueron reportados con sus madres y esto conduciría a sesgos en los resultados, los diferenciales que se observan tanto en los paneles del cuadro como en los de la gráfica son tan marcados y consistentes que permiten confirmar el hecho registrado en

24 Criterio utilizado por las Naciones Unidas en su análisis de las tendencias en la población urbana durante el siglo $\mathrm{XX}^{\prime}$ (The United Nations, Growt of the World's Urban Populations, (1969), Tuble 8. 


\section{Cuadro 8}

MÉXICO: ALGUNOS FACTORES ASOCIADOS CON LA MORTALIDAD INFANTIL, CONDICIÓN DE SUPERVIVENCIA POR RESIDENCIA RURAL O URBANA

\begin{tabular}{|c|c|c|c|c|c|}
\hline \multirow{3}{*}{ Defunciones infantiles } & \multicolumn{2}{|l|}{$\begin{array}{c}\text { Clave de } \\
\text { identificación }\end{array}$} & \multicolumn{3}{|c|}{$\begin{array}{c}\text { Cohorte de nacimientos } 1970-1975 \\
\text { Localidad de residencia }\end{array}$} \\
\hline & & & & & \\
\hline & $(0+1)$ & $\stackrel{\%}{\mathrm{n}}$ & $\begin{array}{c}7.8 \\
(341)\end{array}$ & $\begin{array}{c}5.6 \\
(191)\end{array}$ & $\begin{array}{c}6.8 \\
(532)\end{array}$ \\
\hline Mortalidad neonatal & (0) & $\stackrel{\%}{\mathbf{N}}$ & $\begin{array}{c}4.0 \\
(174)\end{array}$ & $\begin{array}{c}2.9 \\
(100)\end{array}$ & $\begin{array}{l}3.5 \\
(274)\end{array}$ \\
\hline Mortalidad postneonatal & (1) & $\stackrel{\%}{\mathrm{~N}}$ & $\begin{array}{c}3.8 \\
(167)\end{array}$ & $\begin{array}{l}2.7 \\
(91)\end{array}$ & $\begin{array}{l}3.3 \\
(258)\end{array}$ \\
\hline $\begin{array}{l}\text { Sobrevivientes hasta el } \\
\text { primer año de vida }\end{array}$ & (2) & $\stackrel{\%}{\mathrm{~N}}$ & $\begin{array}{c}92.2 \\
(4031)\end{array}$ & $\begin{array}{c}94.4 \\
(3232)\end{array}$ & $\begin{array}{c}93.2 \\
(7263)\end{array}$ \\
\hline $\begin{array}{l}\text { Total de nacidos vivos } \\
\text { considerados }\end{array}$ & Total & & $\begin{array}{l}100.0 \\
(4372)\end{array}$ & $\begin{array}{l}100.0 \\
(3423)\end{array}$ & $\begin{array}{l}100.0 \\
(7795)\end{array}$ \\
\hline
\end{tabular}

$\%$ Distribución porcentual.

N Número de casos.

FUENTE: Encuesta Mexicana de Fecundidad 1976.

la estadística respecto de que en México la mortalidad infantil en las localidades rurales es mayor que en las urbanas. Este diferencial se relaciona con el hecho de que el país cuenta con miles de pequeñas comunidades $\mathrm{y}$, muchas de ellas, están caracterizadas por bajos niveles educativos, condiciones sanitarias poco satisfactorias y deficientes medios para la prevención y el tratamiento de las enfermeda. des. Tal vez de mayor trascendencia que la aserción anterior es advertir que, a lo largo del tiempo, las zonas urbanas registran mayores ganancias en la supervivencia infantil que las zonas rurales y en consecuencia, el diferencial rural-urbano se ha acentuado. Así, para la cohorte 1952-1956, la mortalidad infantil en las zonas urbanas es menor que en las rurales en $18.9 \%$; diez años más tarde (cohorte 1962-1966) en $27.8 \%$ y en el más reciente período estudiado (cohorte 19721976), in $28.3 \%$.

\section{Edad de la madre al dar a luz}

La edad de la madre es uno de los factores que con mayor frecuencia ha sido vinculado con la mortalidad infantil; en general se ha observado que tanto la mortalidad neonatal como la postneonatal aumentan con la edad de la madre. ${ }^{25}$

En el cuadro 9 se advierte que el mayor riesgo de defunción infantil y, la mortalidad neonatal muy superior a la postneonatal, se encuentran en niños nacidos de madres menores de 15 años. Aun cuando el número de nacidos vivos de estas madres que podrían calificarse como demasiado jóvenes, no es alto, es fundamental subrayar los dos hechos anteriores.

A partir de los 15 años de edad al dar a luz, las proporciones de muertes in-

25 Puffer, R. et. al., op. ctt. 


\title{
Gráfica 3
}

\author{
MÉXICO: CONDICIÓN DE SUPERVIVENCIA POR EDAD DE LA MADRE AL DAR \\ A LUZ, COHORTE DE NACIMIENTOS 1970-1975
}

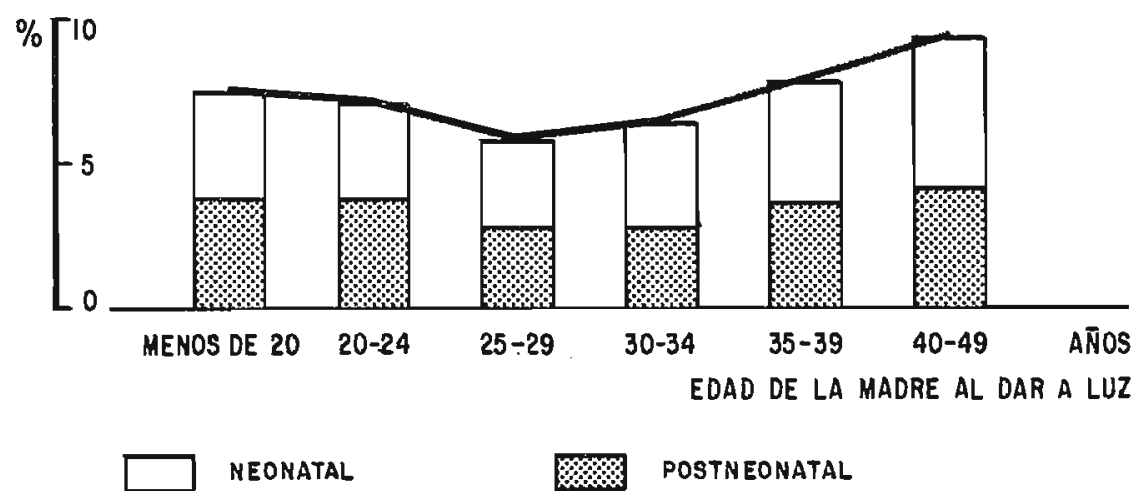

Fuente: Cuodro 9

fantiles presentan una tendencia descendente; alcanzan los valores más bajos y cierta estabilidad cuando la madre tiene entre 25 y 34 años y posteriormente registran una ligera tendencia ascendente. Para obtener una visión más clara al respecto, los resultados se ilustran en la gráfica 3 .

\section{Escolaridad de la madre}

Numerosos trabajos realizados en países con diferentes niveles de desarrollo presentan evidencia de que la educación de la madre juega un papel muy importante en la mortalidad infartil. Por ejemplo, un estudio llevado a cabo en Estados Unidos reportó una tasa de mortalidad infantil de 35 por mil, entre madres con educación elemental comparada con la de 20 por mil entre madres con 40 más años de colegio. ${ }^{26}$ Por otro lado, Caldwell en su análisis de la mortalidad en Nigeria menciona varios estudios en los cuales la relación inversa escolaridad-mortali-

26 Chease, Helen C. and Freida G. Nelson, "Education of mother, medical care and condition of infant", in Helen C. Chease, ed. "A Study of Risks, Medical Care, and Infant Mortality, American Journal of Public Health, Vol. 63, (September 1973), Suplemento, 0. 34. 
dad infantil ha sido encontrada y concluye que en Nigeria la proporción de niños fallecidos disminuye a medida que aumenta la educación. Sus datos también ponen de manifiesto que el diferencial rural-urbano disminuye cuando se controla la educación de la madre. ${ }^{27}$

En la Encuesta Mexicana de Fecundidad, el 18\% de las mujeres entrevistadas declaró que no sabía leer y escribir. Como era de esperarse, el porcentaje de analfabetismo es mayor a medida que la edad aumenta; es así que de las mujeres entre 20 y 24 años sólo el $9.8 \%$ son analfabetas, mientras que para el grupo 45.49 años la cifra alcanza el $32.6 \%$. También se observa que el analfabetismo disminuye a medida que aumenta el tamaño de localidad, (en las localidades de menos de 2500 habitantes es del $31 \%$ y en las localidades de 500000 y más habitantes es del $8 \%$ ).

Respecto al nivel de escolaridad, el $20.2 \%$ reportó nunca haber asistido a la escuela, el $43.7 \%$ tener primaria incompleta; el $15.5 \%$ primaria completa $y$, con estudios superiores a la primaria, el $20.6 \%{ }^{28}$

Aunque el nivel de escolaridad es bajo, las diferencias que se registran por edad de las mujeres muestran el cambio social que se ha venido registrando en el país en las últimas décadas.

En el cuadro 10 aparece la condición de supervivencia por nivel de esolaridad de las madres. En él se observa que, por lo general, los descensos en la mortalidad infantil son constantes al pasar de un nivel de escolaridad al siguiente. Las cifras anteriores son reveladoras del importante papel que juega la educación en la mortalidad infantil. A este respecto se puede conjettirar que las mujeres con educación, además de tener conocimiento acerca de precauciones sanitarias y contar con información nutricional, buscan más activamente la atención médica que aquéllas sin estudios.

Respecto al diferencial rural-urbano y su conexión con la escolaridad de la madre, si fijamos nuestra atención en el cuadro 11 podremos observar, que las cifras correspondientes a la mortalidad infantil así como de sus componentes neonatal, y postneonatal de las localidades urbanas, son inferiores a las de las rurales en 19.1, 18.4 y $19.8 \%$ respectivamente. Sin embargo, cuando se controla la escolaridad de la madre estas diferencias porcentuales disminuyen, sobre todo en los niveles más bajos de escolaridad. Obsérvese por ejemplo que las madres que no tienen escolaridad alguna ya sea que vivan en zonas rurales o urbanas presentan niveles de mortalidad infantil bastante cercanos. La mortalidad neonatal de las que viven en zonas rurales es sólo $1.4 \%$ más baja que la de las que viven en zonas urbanas y el nivel de la mortalidad postneonatal es $0.38 \%$ más alto.

Aun cuando las facilidades de asistencia médica son muy diferentes en ambas localidades, el hallazgo anterior resalta la importancia que reviste la escolaridad

27 Caldwell, J. C. Education as a factor in mortality decline An examination of Nigerian Data, World Health Organization Meeling on Socioeconomic Determinants and Consequences of Mortality. Mexico City, 19-25, June 1979.

28 Los porcentajes relacionados con la escolaridad de las mujeres fueron calculados conforme a los resultados de la Encuesta Mexicana de Fecundidad publicados en el Volumen Il del Primer Informe Nacional. Coordinación General del Sistema Nacional de Información, Secretaría de Programación y Presupuesto, México, marzo 1979, pp. 53-64. 
Cuadro 9

MÉXICO: ALGUNOS FACTORES ASOCIADOS CON LA MORTALIDAD INFANTIL, CONDICIÓN DE SUPERVIVENCIA INFANTIL POR EDAD DE LA MADRE AL DAR A LUZ

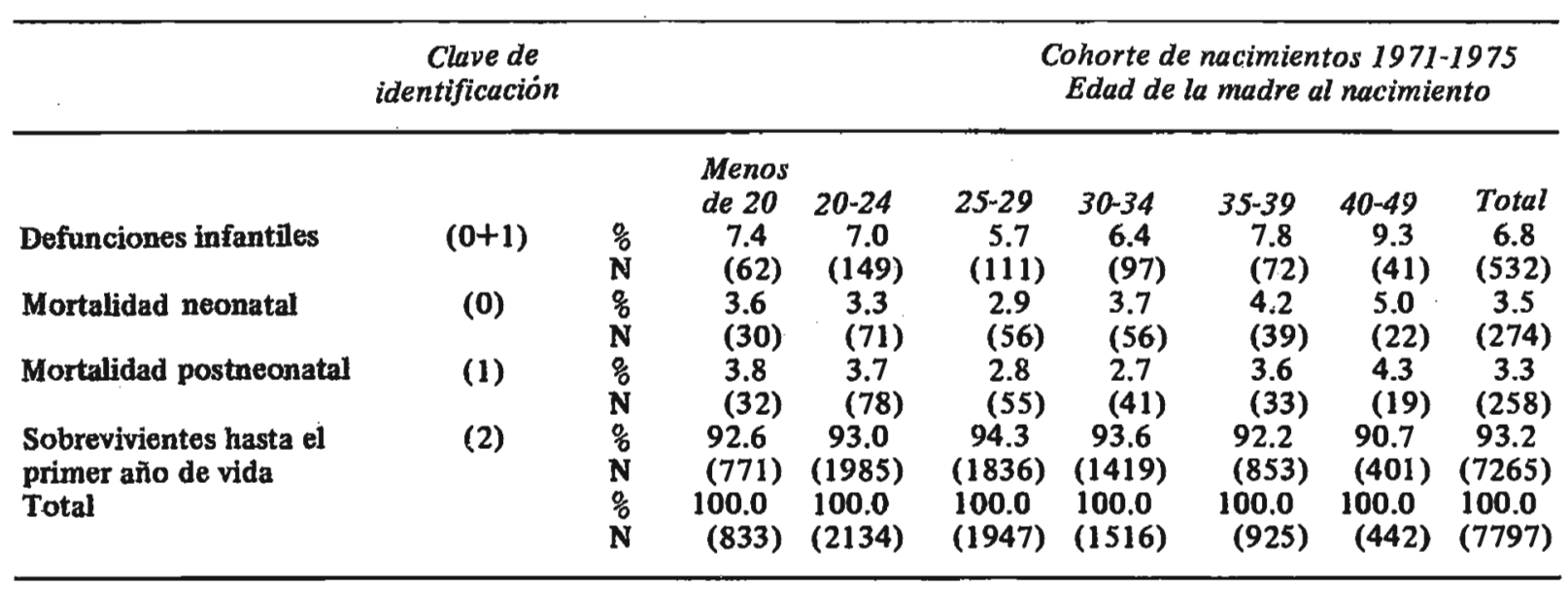

\% Porcentaje respecto al total considerado en cada grupo de edad. N Número de casos.

FUENTE: Encuesta Mexicana de Fecundidad 1976. 
Cuadro 10

MÉXICO: AlgUNOS FACTORES ASOCIADOS CON LA MORTALIDAD INFANTIL, CONDICIÓN DE SUPERVIVENCIA POR ESCOLARIDAD DE LA MADRE

\begin{tabular}{|c|c|c|c|c|c|}
\hline & \multirow[t]{2}{*}{$\begin{array}{c}\text { Clave de } \\
\text { identificación }\end{array}$} & & \multicolumn{2}{|c|}{$\begin{array}{c}\text { Cohorte de nacimientos 1970-1975 } \\
\text { Es colaridad }\end{array}$} & \multirow[b]{2}{*}{ Total } \\
\hline & & & $\begin{array}{l}\text { Ninguna } y \\
\text { Primaria } \\
I^{\circ}, 2^{\circ}, 3^{\circ}\end{array}$ & $\begin{array}{l}\text { Primaria } \\
4^{\circ}, 5^{\circ}, 6^{\circ} \\
y+\end{array}$ & \\
\hline Defunciones infantiles & $(0+1)$ & $\stackrel{\%}{\mathbf{N}}$ & $\begin{array}{c}7.8 \\
(370)\end{array}$ & $\begin{array}{c}5.3 \\
(162)\end{array}$ & $\begin{array}{r}6.8 \\
(532)\end{array}$ \\
\hline Mortalidad neonatal & (0) & $\frac{\%}{N}$ & $\begin{array}{c}3.9 \\
(185)\end{array}$ & $\begin{array}{l}2.9 \\
(89)\end{array}$ & $\begin{array}{r}3.5 \\
(274)\end{array}$ \\
\hline Mortalidad postneonatal & (1) & $\%$ & $\begin{array}{l}3.9 \\
(185)\end{array}$ & $\begin{array}{l}2.4 \\
(73)\end{array}$ & $\begin{array}{r}3.3 \\
(258)\end{array}$ \\
\hline $\begin{array}{l}\text { Sobrevivientes hasta el } \\
\text { primer año de vida }\end{array}$ & (2) & $\stackrel{\%}{\mathbf{N}}$ & $\begin{array}{c}92.2 \\
(4385)\end{array}$ & $\begin{array}{c}94.7 \\
(2878)\end{array}$ & $\begin{array}{r}93.2 \\
(7263)\end{array}$ \\
\hline $\begin{array}{l}\text { Total de nacidos vivos } \\
\text { considerados }\end{array}$ & Total & $\stackrel{\%}{\mathbf{N}}$ & $\begin{array}{l}100.0 \\
(4755)\end{array}$ & $\begin{array}{l}100.0 \\
(3040)\end{array}$ & $\begin{array}{r}100.0 \\
(7795)\end{array}$ \\
\hline
\end{tabular}

\% Distribución porcentual.

N Número de casos.

FUENTE: Encuesta Mexicana de Fecundidad 1976. 
Cuadro 11

MÉXICO: ALGUNOS FACTORES ASOCIADOS CON LA MORTALIDAD INFANTIL, CONDICIÓN DE SUPERVIVENCIA POR ESCOLARIDAD DE LA MADRE

\begin{tabular}{|c|c|c|c|c|c|c|c|c|}
\hline & \multirow{2}{*}{\multicolumn{2}{|c|}{$\begin{array}{c}\text { Clave de } \\
\text { identificación }\end{array}$}} & \multirow[b]{2}{*}{ Ninguna } & \multirow[b]{2}{*}{$\begin{array}{l}\text { Prima- } \\
\text { ria } \\
1^{\circ}, 2^{\circ}, 3^{\circ}\end{array}$} & \multicolumn{4}{|c|}{$\begin{array}{c}\text { Cohorte de nacimientos 1971-1975 } \\
\text { Es colaridad }\end{array}$} \\
\hline & & & & & $\begin{array}{l}\text { Prima- } \\
\text { ria } \\
4^{\circ}, 5^{\circ}, 6^{\circ},\end{array}$ & $\begin{array}{c}\text { Secun- } \\
\text { daria } \\
\text { incom- } \\
\text { pleta }\end{array}$ & $\begin{array}{c}\text { secun- } \\
\text { daria } \\
\text { comple- } \\
\text { ta } y+\end{array}$ & Total \\
\hline Defunciones infantiles & $(0+1)$ & $\begin{array}{l}\% \\
\mathbf{N}\end{array}$ & $\begin{array}{c}8.0 \\
(150)\end{array}$ & $\begin{array}{c}7.7 \\
(220)\end{array}$ & $\begin{array}{c}5.4 \\
(112)\end{array}$ & $\begin{array}{l}5.7 \\
(20)\end{array}$ & $\begin{array}{l}5.0 \\
(30)\end{array}$ & $\begin{array}{r}6.8 \\
(532)\end{array}$ \\
\hline Mortalidad neonatal & (0) & $\stackrel{\%}{\mathbf{N}}$ & $\begin{array}{l}4.2 \\
(78)\end{array}$ & $\begin{array}{c}3.7 \\
(107)\end{array}$ & $\begin{array}{l}2.6 \\
(53)\end{array}$ & $\begin{array}{l}3.4^{*} \\
(12)\end{array}$ & $\begin{array}{l}4.0 \\
(24)\end{array}$ & $\begin{array}{r}3.5 \\
(274)\end{array}$ \\
\hline Mortalidad postneonatal & (1) & $\%$ & $\begin{array}{l}3.8 \\
(72)\end{array}$ & $\begin{array}{c}4.0 \\
(173)\end{array}$ & $\begin{array}{l}2.8 \\
(59)\end{array}$ & $\begin{array}{r}2.3^{*} \\
(8)\end{array}$ & $\begin{array}{r}1.0^{*} \\
(6)\end{array}$ & $\begin{array}{r}3.3 \\
(258)\end{array}$ \\
\hline $\begin{array}{l}\text { Sobrevivientes hasta el } \\
\text { primer año de vida }\end{array}$ & (2) & $\stackrel{\%}{\mathrm{~N}}$ & $\begin{array}{c}92.0 \\
(1730)\end{array}$ & $\begin{array}{c}92.3 \\
(2655)\end{array}$ & $\begin{array}{c}94.6 \\
(1971)\end{array}$ & $\begin{array}{l}94.3 \\
(333)\end{array}$ & $\begin{array}{l}95.0 \\
(574)\end{array}$ & $\begin{array}{r}93.2 \\
(7263)\end{array}$ \\
\hline $\begin{array}{l}\text { Total de nacidos vivos } \\
\text { considerados }\end{array}$ & Total & $\stackrel{\%}{\mathrm{~N}}$ & $\begin{array}{l}100.0 \\
(1880)\end{array}$ & $\begin{array}{l}100.0 \\
(2875)\end{array}$ & $\begin{array}{l}100.0 \\
(2083)\end{array}$ & $\begin{array}{r}100.0 \\
(353)\end{array}$ & $\begin{array}{c}100.0 \\
\cdot \quad(604)\end{array}$ & $\begin{array}{r}100.0 \\
(7795)\end{array}$ \\
\hline
\end{tabular}

\% Distribución porcentual.

N Número de casos.

* Menos de 15 casos.

FUENTE: Encuesta Mexicana de Fecundidad 1976. 
en comparación con la residencia rural-urbana, en el riesgo de mortalidad tanto neonatal como postneonatal.

Del análisis de las tabulaciones anteriores, a manera de resumen puede destacarse lo siguiente: se observó que la mortalidad infantil es más alta cuando: el niño pertenece al sexo masculino, reside en localidades rurales, no es alimentado con leche materna y cuando los intervalos entre nacimientos sucesivos son menores de un año. También existen mayores probabilidades de muerte infantil cuando la madre es muy joven, ha sufrido previas pérdidas reproductivas o carece de escolaridad. Este último factor juega un papel muy importante en la supervivencia infantil por un lado, el riesgo de muerte infantil desciende a medida que aumentan los años de estudio, y por otro, el diferencial nural-urbano disminuye cuando se controla el nivel de escolaridad de la madre.

\section{ANÁLISIS ESTADISTICO}

Hasta aqui se ha analizado las relaciones entre las variables pero no se ha evaluado la importancia que cada variable tiene en la mortalidad infantil. Se plantea, por lo tanto, la necesidad de efectuar un análisis que permita observar la influencia que, sobre la variable dependiente, ejerce cada una de las variables independientes. La forma de lograr este objetivo y al mismo tiempo ser consistentes con el nivel de análisis que se ha mantenido hasta ahora, ha sido aplicar a los datos un modelo de regresión múltiple con variables rudas ("dummy variable").

Somos conscientes de que, al limitar los valores de las variables independientes a sólo dos posibilidades se perderá información valiosa. Por lo tanto, al contemplar los resultados finales, habría que tener en consideración las diversas categorias que esconden las agrupaciones realizadas.

En base a los principales cambios registrados en las tabulaciones cruzadas, los valores de las variables independientes en el estudio fueron recodificadas para que estas variables fueran convertidas en mudas. La transformación se efectuó asignándoles los valores 0 o 1 conforme a las características que a continuación se señalan:

1. Edad de la madre al nacimiento (Variable $X_{2}, X_{3}$ )

$$
\text { Valores }\left\{\begin{array}{lll}
\frac{X_{2}}{0} & X_{3} & \\
0 & 0 & \begin{array}{l}
\text { Si la madre tenía, al nacimien } \\
\text { menos de } 20 \text { años. }
\end{array} \\
1 & 0 & \text { Si tenía entre } 20 \text { y } 34 \text { años. } \\
0 & 1 & \text { Si tenía } 35 \text { años o más }
\end{array}\right.
$$

2. Intervalo entre el nacimiento observado y el anterior (Variable $X_{4}$ )

$$
X_{4}=\begin{array}{ll}
0 & \text { intervalo menor de } 12 \text { meses } \\
1 & \text { intervalo mayor de } 12 \text { meses }
\end{array}
$$


3. Existencia de lactancia (Variable $X_{5}$ )

$$
X_{5}=\begin{array}{ll}
0 & \text { No fue alimentado con leche materna. } \\
1 & \text { Si fue alimentado con leche materna. }
\end{array}
$$

4. Existencia de previas pérdidas reproductivas (Variable $X_{6}$ )

$$
\begin{aligned}
& X_{6}=0 \begin{array}{l}
\text { No acontecieron previas pérdidas repro- } \\
\text { ductivas }
\end{array} \\
& 1 \text { Si acontecieron previas pérdidas repro- } \\
& \text { ductivas. }
\end{aligned}
$$

5. Escolaridad de la madre (Variable $X_{7}$ )

$$
X_{7}=\begin{array}{ll}
0 & \text { Analfabeta. Ninguin año de escuela. } \\
1 & \text { Alfabeta. Uno o más años de escuela. }
\end{array}
$$

Una vez transformadas las variables independientes en variables mudas, se determinaron todas las posibles combinaciones de sus valores. El número total de combinaciones resultó ser de 48 , puesto que la variable "edad de la madre al dar a luz" tenía tres posibilidades y, escolaridad, pérdidas previas, lactancia e intervalo intergenésico tenían dos cada una. La matriz de variables mudas quedó integrada con 48 renglones y 6 columnas (ver cuadro 12).

Para encontrar dentro del total de nacidos vivos para cada una de las $48 \mathrm{com}$ binaciones, el número de sobrevivientes a los 12 meses, el número de muertes entre 1 y 11 meses y el número de muertes de menores de un mes así como el total de nacidos vivos, se efectuó el cruce simultáneo de las cinco variables independientes. Con estas cifras se calcularon, para cada renglón, las tasas de sobrevivencia infantil (SI), neonatal (SN) y postneonatal (SP), en la forma siguiente:

SI

$$
\begin{aligned}
& =\frac{\text { número de sobrevivientes hasta un año }}{\text { total de nacidos vivos }} \\
& \frac{\text { número de sobrevivientes hasta un mes }}{\text { total de nacidos vivos }}
\end{aligned}
$$

$\mathrm{C} 2-\mathrm{CO}$

$S P$

total de sobrevivientes entre 1 y 11 meses total de nacidos vivos

LOS vectores $S I, S N$, y $S P$ con las tasas de sobrevivencia infantil, neonatal y postneonatal para cada renglón, se presentan en el cuadro 12.

A cada vector de las tasas de sobrevivencia le fue aplicado el siguiente modelo:

$$
Y=B_{1}+B_{2} X_{2}+B_{3} X_{3}+B_{4} X_{4}+B_{5} X_{5}+B_{6} X_{6}+B_{7} X_{7}+\ell
$$

donde:

$Y=$ Tasa de sobrevivencia (infantil, neonatal o postneonatal) en un renglón. 
$B_{1} \quad$ Nivel de $Y$ para la situación; madres menores de 20 años, intervalo intergenésico menor de 12 meses; sin lactancia, sin previas pérdidas repro. ductivas y sin escolaridad.

$B_{2}=$ Efecto sobre $Y$ de la edad de la madre cuando ésta está comprendida entre los 20 y 34 años.

$B_{3}=$ Efecto sobre $Y$ de la edad de la madre cuando ésta es mayor de 35 años de edad.

$B_{4}=$ Efecto sobre $Y$ de un intervalo entre nacimientos mayor de 12 meses.

$B_{5}=$ Efecto sobre $Y$ de la lactancia.

$B_{6}=$ Efecto sobre $Y$ de la existencia de previas pérdidas reproductivas.

$B_{7}=$ Efecto sobre $Y$ de la escolaridad de la madre.

\section{RESULTADO DE LAS REGRESIONES}

En el cuadro 12 aparecen los resultados obtenidos en las regresiones. Puede observarse que para cada condición de sobrevivencia se presentan tres ecuaciones: la primera contiene las 5 variables independientes mencionadas; la segunda no incluye lactancia y la tercera no incluye intervalo intergenésico. Vale la pena hacer notar que dado que la variable $X_{5}$ (intervalo intergenésico) no existe para todos los nacidos vivos, cuando participa en la regresión, los nacimientos de primer orden son eliminados y los resultados sólo son aplicables a los nacimientos del segundo orden en adelante.

Considerando posibles derivados ya sea de los datos o bien de la conversión a "variables mudas", las variables en conjunto explican para cada caso, un buen porcentaje de la varianza en la condición de sobrevivencia infantil. Las constantes representan el nivel de las tasas de supervivencia para la situación: madres menores de 20 años, intervalo intergenésico menor de 12 meses, ausencia de lactancia, ausencia de pérdidas reproductivas previas y madres sin escolaridad.

En las regresiones puede observarse que los coeficientes más altos y, consecuentemente, una importante contribución en $R^{2}$ pertenecen a las variables $X_{5}$ (lactancia) $X_{4}$ (intervalo intergenésico) $X_{6}$ (previas pérdidas reproductivas) y $X_{7}$ (educación de la madre). Esto significa que una mayor supervivencia y, por lo tanto, menor mortalidad infantil, está asociada con las siguientes condiciones: intervalo intergenésico mayor de 12 meses, alimentación con leche materna, educación de la madre y ausencia de pérdidas reproductivas.

Al analizar por separado la sobrevivencia neonatal y postneonatal, aunque los resultados aparecen bastante consistentes en cuanto a la importancia de las variables, se observa que los coeficientes en la postneonatal son por lo general más altos que los de la neonatal y que, además, explican un mayor porcentaje de la varianza. 


\section{Cuadro 12}

MÉXICO: ALgUNOS FACTORES ASOCIADOS CON LA MORTALIDAD INFANTIL, COHORTE DE NACIMIENTOS 1970-1975, RESULTADOS DE REGRESION

\begin{tabular}{lcc}
\hline Edad de la madre & $\begin{array}{c}\text { Intervalo } \\
\text { interge- } \\
\text { nésico }\end{array}$ & $\begin{array}{c}\text { Lactancias } \\
\text { pérdidas Escolaridaq } \\
\text { reproductivas }\end{array}$ \\
\hline
\end{tabular}

Supervivencia infantil

1) Excluyendo el primer orden de nacimiento en la cohorte.

$\mathrm{R}^{2}=0.3795$

a) $\mathrm{S} 1=0.702+0.004 \mathrm{X}_{2}-0.012 \mathrm{X}_{3}+0.080 \mathrm{X}_{4}+0.210 \mathrm{X}_{5}-0.053 \mathrm{X}_{6}+.0 .037 \mathrm{X}_{7}$

$\mathrm{R}^{2}=0.2434$

b) $\mathrm{SI}=0.884+0.011 \mathrm{X}_{-2}^{*}+0.014_{3}^{*}+0.084 \mathrm{X}_{4} \quad-0.061 \mathrm{X}_{6}+0.025 \mathrm{X}^{*} \eta$

Para todos los nacidos vivos en la cohorte.

$\mathrm{R}^{2}=0.7704$

SI $=0.758+0.014 \mathrm{X}_{2}+0.020 \mathrm{X}_{3}+0.187 \mathrm{X}_{5}-0.016 \mathrm{X}_{6}+0.044 \mathrm{X}_{7}$

Supervivencla neonatal

1) Excluyendo el primer orden de nacimiento en la cohorte.

$\mathrm{R}^{2}=0.5774$

a) $\mathrm{SN}=0.807+0.024 \mathrm{X}_{2}-0.007 \mathrm{X}_{3}+0.034 \mathrm{X}_{4}+0.191 \mathrm{X}_{5}-0.042 \mathrm{X}_{6}+0.034 \mathrm{X}_{2}$

$\mathrm{R}^{2}=0.2720$

b) $\mathrm{SN}=0.950+0.027 \mathrm{X}^{*}{ }_{2}-0.005 \mathrm{X}^{*}{ }_{3}+0.049 \mathrm{X}_{4} \quad 0.044 \mathrm{X}_{6}+0.015 \mathrm{X}^{*}$,

Para todos los nacidos vivos en la cohorte.

$\mathrm{R}^{2}=0.6090$

$\mathrm{SN}=0.825+0.004 \mathrm{X}_{2}+0.008 \mathrm{X}_{3} \quad+0.162 \mathrm{X}_{5}-0.017 \mathrm{X}_{6}+0.033 \mathrm{X}_{7}$

Supervivencia postneonatal.

1) Excluyendo el primer orden de nacimiento en la cohorte.

$\mathrm{R}^{2}=0.4908$

a) $\mathrm{SP}=0.509+0.021 \mathrm{X}_{2}-0.004 \mathrm{X}_{3}+0.114 \mathrm{X}_{4}+0.400 \mathrm{X}_{5}-0.095 \mathrm{X}_{6}+0.071 \mathrm{X}_{7}$

$\mathrm{R}^{2}=0.2687$

b) $\mathrm{SP}=0.834+0.038 \mathrm{X}^{*}+0.019 \mathrm{X}^{*}{ }_{3}+0.128 \mathrm{X}_{4} \quad-0.110 \mathrm{X}_{6}+0.039 \mathrm{X}^{*} 7$

Para todos los nacidos vivos en la cohorte.

$\mathrm{R}^{2}=0.7926$

$\mathrm{SP}=0.583+0.018 \mathrm{X}_{2}{ }_{2}+0.028 \mathrm{X}_{3} \quad+0.349 \mathrm{X}_{3}-0.033 \mathrm{X}_{6}+0.077 \mathrm{X}_{7}$

* Estadísticamente no significativa al nivel del $95 \%$.

a) La ecuación de regresión incluye la variable lactancia.

b) La ecuación de regresión no incluye la variable lactancia.

FUENTE: Encuesta Mexicana de Fecundidad, 1976. 
Finalmente, en la regresión también se observa que los coeficientes de la madre al dar a luz y educación de la madre, presentan valores pequeños y estadísticamente no significativos. Respecto a la escolaridad de la madre, cabe recordar que en las tablas cruzadas que se presentaron en páginas anteriores se observó claramente que a medida que aumenta el nivel educativo de la madre aumenta la supervivencia infantil. Es muy probable que en este análisis, al darle sólo dos posibilidades (menos de 4 años y cuatro años o más de asistencia escolar), la influencia de la educación de la madre sobre la mortalidad infantil no se perciba con claridad.

De acuerdo con lo anterior puede concluirse que las mejores condiciones para que un nirio logre alcanzar su primer año de vida se encuentran cuando la madre es mayor de 24 años y menor de 35, el intervalo entre nacimientos es más largo que 12 meses, el niño es alimentado con leche materna, la madre no ha sufrido previas pérdidas reproductivas y por lo menos llegó hasta el cuarto grado de primaria. Por el contrario, la maternidad a edades cercanas al principio y final de la etapa reproductiva, los cortos intervalos entre nacimientos, la ausencia de lactancia, las pérdidas reproductivas y la nula escolaridad de la madre, son factores que están asociados a un mayor riesgo de que el niño muera antes de cumplir su primer aniversario.

Las aseveraciones anteriores son válidas para todo el primer año de vida de los infantes, sin embargo, la influencia de las variables estudiadas es mucho mayor en el período postneonatal que en el neonatal.

\section{ANÁLISIS SOBRE MORTALIDAD INFANTIL DEL ÚlTIMO NACDO VIVO}

Con el objeto de realizar un análisis que en vez de la conversión èn variables mudas, utilizara los diferentes valores de las variables independientes, $\mathrm{y}$ al mismo tiempo incorporar nuevos elementos en la regresión, se seleccionó como variable dependiente la condición de sobrevivencia del último nacido vivo de cada mujer. ${ }^{29}$ Además de las cinco variables independientes incluidas en el análisis del total de nacidos vivos, se añadieron las 7 siguientes: orden de nacimiento, tamaño de la localidad de residencia, grado de hacinamiento; ${ }^{30}$ ingreso mensual por persona en el hogar; ${ }^{31}$ indice del nivel de vida; ${ }^{32}$ abastecimiento de agua entubada y disponibilidad de servicio higiénico con excusado. ${ }^{33}$

29 Sólo se tomaron en cuenta los nacimientos del último orden ocurridos durante los dioz años anteriores a la encuesta, es decir, a partir de 1967.

30 Grado de hacinamiento. Representa el número de residentes por habitación. Se calculó únicamente para las viviendas que contienen un solo hogar.

31 Ingreso mensual por persona en el hogar. Se obturo mediante la suma de los ingresos de la entrevistada (si trabaja y recibe un salario por sus servicios) y de su esposo, dividida en. tre el número de miembros en el hogar.

32 Este índice va de 0 a 15 y se construyó con la información sobre el material de que está hecha la vivienda: (muros, pisos y techos), la existencia de electricidad y disponibilidad de aparatos como estufa, plancha, refrigerador, televisión y radio.

33 Las variables disponibilidad de agua y de servicios higiénicos con excusado, son dicotómicas. 
Los resultados de la regresión se presentan enseguida:

\begin{tabular}{lcc}
\hline$R^{2}=0.1774$ & \multicolumn{2}{c}{ Coeficiente } \\
\hline Variables dependientes & No estandarizados & Estandarizados \\
\hline Orden de nacimiento & $-0.005^{*}$ & $-0.076^{*}$ \\
Número de previas pérdidas & -0.014 & -0.062 \\
reproductivas & $-0.000^{*}$ & $-0.016^{*}$ \\
Edad de la madre al nacimientc & 0.053 & 0.087 \\
Tamaño de localidad & 0.089 & 0.219 \\
Años de estudio de la madre & 0.032 & 0.325 \\
Tiempo de lactancia & 0.026 & 0.061 \\
Disponibilidad de agua & & 0.063 \\
Disponibilidad de servicio & 0.024 & \\
higiénico & 1.852 & \\
Constante & & \\
Número de casos incluidos 2 769 & &
\end{tabular}

* Estadísticamente no significativo al $95 \%$ según la prueba $F$.

De las cifras anteriores podemos derivar que los años de estudio de la madre, el tiempo de lactancia, el nivel de bienestar de la familia, el abastecimiento de agua potable y la disponibilidad de servicio higiénico con excusado, están positivamente relacionados con la sobrevivencia infantil, mientras que el número de previas pérdidas reproductivas y el orden de nacimientos lo están negativamente. Por otro lado, en la ecuación de regresión la variable edad de la madre al dar a luz, no parece ser estadísticamente significativa.

Si bien es cierto que las variables en conjunto sólo explican el $17 \%$ de la varianza, en análisis precedente, además de confirmar los hallazgos anteriores, destaca la importancia que en la sobrevivencia infantil tienen tanto la educación de la madre, como la disponibiliclad de agua y servicio higiénico en la vivienda.

\section{CONCLUSIONES Y RECOMENDACIONES}

La mortalidad infantil está en gran medida asociada con factores que no ha sido posible incorporar en el análisis. Como resultado de la investigación podemos decir que dentro de las variables incluidas en el estudio, los más altos riesgos de muerte, tanto infantil como entre 1 y 5 años, se encuentran cuando el intervalo que media entre dos nacimientos sucesivos es menor que un año. Por lo general a medida que e] intervalo intergenésico aumenta, el riesgo de mortalidad disminuye, sin embargo esta relación inversa se convierte en directa cuando el tiempo transcurrido entre dos nacidos vivos es superior a los 4 años.

Los menores indices de mortalidad infantil se registran cuando la madre no ha sufrido con anterioridad pérdidas reproductivas. Por otro lado si la mujer tiene en su pasado muertes infantiles o embarazos terminados en aborto o nacido muerto, las probabilidades de ocurrencia de muerte infantil son mayores. También 
se observó que a medida que aumenta el número de previas pérdidas aumenta también la mortalidad infantil.

Los porcentajes de mortalidad neonatal, postneonatal y consecuentemente infantil, son más altos para los niños que no fueron alimentados con leche materna que para aquéllos que si lo fueron.

La mortalidad infantil en las localidades rurales es mayor que en las urbanas, y a lo largo del tiempo la brecha en vez de reducirse se ha ampliado.

Los más altos riesgos de defunciones infantiles y mortalidad neonatal muy superior a la postneonatal, se encuentran en niños nacidos de madres menores de 15 años. A partir de los 15 años de edad al dar a luz las proporciones de muertes infantiles presentan una tendencia descendente; alcanzan los valores más bajos y cierta estabilidad cuando la madre tiene entre 25 y 34 años y posteriormente registran una ligera tendencia ascendente.

La educación de la madre juega un papel muy importante en la mortalidad infantil. Se observaron constantes descensos en la mortalidad infantil al pasar de un nivel de escolaridad al siguiente. Por otra parte, cuando se controla la escolaridad de la madre, el diferencial rural-urbano disminuye sobre todo en los niveles más bajos de escolaridad. Dado que las facilidades de asistencia médica son muy diferentes en ambos tipos de localidad, el hallazgo anterior resalta la relevancia que tiene la escolaridad de la madre en el riesgo de mortalidad en la primera infancia.

Se observó también que el abastecimiento de agua potable y la disponibilidad de servicio higiénico con excusado están positivamente relacionados con la sobrevivencia infantil.

\section{CONSIDERACIONES FINALES}

Algunas muertes en los primeros años de vida son inevitables pero muchas otras pueden ser prevenidas con la medicina moderna, medidas higiénicas y de conservación de la salud $y$, además, mediante la orientación adecuada a las parejas.

En la actualidad, los altos niveles de mortalidad en la temprana infancia no son justificables, por lo tanto, existe una extensa área de acción para conseguir descensos considerables en esta variable demográfica.

Dentro de las apremiantes tareas a realizar en el país, encaminadas a alcanzar este objetivo se pueden mencionar las siguientes:

Extender y mejorar los servicios de salud y atención materno-infantil así como programas de inmunización, sobre todo en las pequeñas comunidades. A este respecto cabe señalar que el derecho de salud es garantía constitucional de todos los mexicanos.

Lanzar campañas de orientación para las mujeres sobre la importancia de la edad de la madre al nacimiento de los hijos, los intervalos entre nacimientos y la alimentación con leche materna.

Otorgar prioridad a programas encaminados a erradicar el analfabetismo y elevar el nivel cultural y socioeconómico de la población con especial énfasis en los grupos menos favorecidos de la escala social. 
Intensificar programas para proveer agua potable, instalaciones de alcantarillado a todas las localidades que carecen de estos servicios indispensables.

En resumen, mediante el análisis de las variables incorporadas en el estudio se ha demostrado que existen condiciones bajo las cuales los niños tienen una mayor probabilidad de sobrevivir. La lucha contra la mortalidad infantil en nuestro país es una tarea apremiante y, para llevarla a cabo exitosamente es necesario multiplicar esfuerzos en diferentes direcciones. Aun cuando extensas campañas de salud pública y de atención y orientación materno-infantil son necesarias, de ninguna manera pueden considerarse como suficientes. Para triunfar en esta empresa será indispensable que ocurran cambios sociales y económicos dentro de la población que se encuentra en los estratos menos favorecidos de la escala social. 